\title{
Medical Aid in Dying, Hastened Death and Suicide: A Qualitative Study of Hospice Professionals' Experiences from Washington State
}

Sheri Mila Gerson, $\mathrm{PhD}^{1,2}$; Nancy Preston, $\mathrm{PhD}^{1}$; Amanda Bingley, $\mathrm{PhD}^{1}$

${ }^{1}$ International Observatory on End of Life Care, Division of Health Research, Lancaster University, Lancaster, United Kingdom

${ }^{2}$ University of Glasgow, Dumfries Campus, School of Interdisciplinary Studies, Scotland, United Kingdom

\section{Corresponding Author:}

Sheri Mila Gerson, PhD, LICSW

Tel: 1(360)789-0738

Email: sherimila@gmail.com

Number of tables: 1

Number of figures: 0

References: 48

Word count (text only): 3492

Running title: Aid in Dying, Hastened Death and Suicide

Financial Disclosures: The authors have no financial relationship relevant to this article to disclose

Potential Conflict of Interest: The authors have no conflict of interest relevant to this article to disclose. 


\title{
Medical Aid in Dying, Hastened Death and Suicide: A Qualitative Study of Hospice Professionals' Experiences from Washington State
}

\begin{abstract}
Context Many jurisdictions around the world have passed medical aid in dying laws allowing competent, eligible individuals facing life-limiting illness to self-administer prescribed medication to control timing of death. These laws do not prevent some patients who are receiving hospice services from dying by suicide without assistance.
\end{abstract}

Objectives To explore hospice professionals' experiences of patients who die by suicide or intentionally hasten death with or without legal assistance in an area where there is legalized medical aid in dying.

Methods Semi-structured in-depth qualitative interviews were conducted with twenty-one home hospice professionals ( 7 nurses, 7 social workers, 4 physicians, 3 chaplains). Thematic analysis was carried out to analyze the data.

Results Three primary themes were identified from the interviews: 1) dealing with and differentiating between hastened death and suicide, 2) medical aid in dying access and affordability, and 3) how patients have hastened their own deaths. Analysis of these data indicates there are some patients receiving hospice services who die by suicide because they are not eligible for, have no knowledge of, or lack access to legalised medical aid in dying. Hospice professionals do not consistently identify patients' deaths as suicide when they are self-inflicted and sometimes view these deaths as justified. 
Conclusion Suicide and hastened deaths continue to be an unexamined cause of death for some home hospice patients who may have requested medical aid in dying. Open communication and increased education and training is needed for palliative care professionals regarding legal options, issues of suicide and suicide assessment.

\section{Keywords}

suicide

assisted suicide

assisted dying

euthanasia

hospice

palliative care 


\section{Introduction}

Many areas around the world including Belgium, Canada, Netherlands, Switzerland, and Victoria, Australia and several US states allow a physician, or in some areas a nurse practitioner, to prescribe a lethal dose of medication for an individual to self-administer $(1,2)$. The US States of California, Colorado, Hawaii, Maine, Montana, New Jersey, Oregon, Vermont, Washington, and the District of Columbia (3-10) have laws allowing eligible adults deemed to be mentally competent, and who have a life-limiting prognosis of six months or less, the option of requesting a self-administered lethal dose of medications prescribed by their physician (11). Medical aid in dying (MAID) is a term becoming more widely used to describe this process in the United States (12). Deaths in locations where MAID is authorized in the United States are not considered suicide if supported by documents regulated by the laws (11). But not all patients who choose to hasten death use MAID and instead die by other self-inflicted methods. Some researchers and professionals may consider MAID to be a form of rational suicide (13-18) for a person seeking assistance to die when facing life limiting illness. Despite these accounts, very little is known about professionals' experiences with hospice patients who die by suicide, particularly in an area that legally permits MAID. A systematic review revealed that more research is needed to understand what hastened death and suicide mean in palliative care in areas where MAID is lawful (19). If patients are dying by suicide without MAID, it is important to understand professionals' experiences of suicide with their patients to improve patient care and safety, and develop hospice and palliative care responses to concerns.

The research focus was to examine professionals' experiences of suicide and hastened death with hospice patients in Washington State. Bostwick and Cohen (20) discuss that a hastened death "examines the individual's intentions with respect to dying and considers the involvement of social networks" that support the wish to die. Social networks, however, may 
not be required to distinguish a hastened death from a suicide. The term 'hastened death' for this study includes both intentional suicide and deaths from MAID. Most research on hastened death was completed in locations where MAID was not legally authorised at the time of the research or focused on the desire for hastened death and not the actual experiences of patients who died by suicide (21-29). The objective of this research was to explore hospice professionals' experiences with patients who die by suicide or intentionally hasten death with or without legal assistance in an area where there is legalized MAID.

\section{Methods}

This study was conducted with home hospice professionals in Washington State, where the Death with Dignity Act was implemented in 2009. Hospice teams in the United States include social workers, nurses, physicians and chaplains who primarily provide consultation and services primarily in home settings regarding pain and symptom management, and support the patient, caregivers and families $(30,31)$. Professionals work for different types of hospice organizations, both religious and non-religious, which have policies about the Death with Dignity Act that may also influence their practice (32).

\section{Recruitment and sample}

Participants in Washington State were purposely recruited through professional organizations, conferences and meetings to participate in this interview study. Purposive recruitment ensured professionals were employed by different types of hospice institutions across diverse areas of the state. This study aimed to explore the subject in-depth and did not seek data saturation. Whenever possible, a face-to-face interview was completed but the option of a phone interview was also offered. The participants were contacted by email or telephone to receive information about the study. The participant was reminded that they had the option to decline to participate, 
to withdraw before or during the interview, or withdraw their data up to two weeks following completion of the interview.

\section{Data acquisition}

Face-to-face or telephone interviews lasting one to two hours, using in-depth, semi-structured and open-ended questions, were conducted in 2015 by SMG. SMG was transparent about previous experience as a hospice professional but had no personal or professional relationship with any of the participants. An interview guide, included in Appendix 1, helped to facilitate questions but interviewing followed an adaptive process to address any new issues raised by participants. Two face-to-face pilot interviews with hospice professionals were conducted to develop and refine interview questions. Not all participants responded to each question and transcripts were not sent to participants for comment. The emotional impact of participating in the study was considered and each participant was offered referral in case of distress, however no request was made.

The protocol was reviewed by the Lancaster University Faculty of Health and Medicine Research Ethics Committee and approved by the Lancaster University Research Ethics Committee, FHMREC14031. All participants provided written informed consent.

\section{Data Analysis}

Thematic analysis with an inductive approach was used to interpret themes extracted from the data and to identify and compare experiences. Thematic analysis is employed widely in health research as a method that is suited to questions about people's experiences focused on developing patterned meanings across the data (33). All interviews were recorded and transcribed by SMG directly into a Word document, then reviewed and reformatted before uploading into QSR NVivo10. Any information that could identify a participant was deleted. Each line was numbered to be able to refer to it as needed and NVivo10 was used to create a 
coding structure, develop new codes and link them to the data sources. Coding was completed by SMG through an iterative process, and re-coded into categories. To improve trustworthiness, three transcripts were reviewed by two researchers on the supervisory team who produced another coding framework. Categories were discussed and reviewed with all researchers until consensus about final themes was achieved. This study utilised Braun and Clarke's (34) 15-point checklist of criteria for good thematic analysis.

\section{Results}

A total of twenty-one interviews were completed with seven nurses, seven social workers, four physicians, and three chaplains. Sixteen were face-to-face interviews and five were completed by telephone. Table 1 identifies information about participants:

$<$ INSERT TABLE $1>$

\section{Themes}

Three primary themes were identified from the interviews: 1) Dealing with and differentiating between hastened death and suicide, 2) MAID access and affordability, and 3) how patients have hastened their own deaths.

\section{1) Dealing with and differentiating between hastened death and suicide}

Distinguishing between deaths from suicide and deaths that were intentionally hastened but not considered suicide was found to be challenging for most of the participants. When asked about experiences with patients who died by suicide or hastened death, all participants spoke about patients who died by suicide or utilized MAID. Overall, the suicides that were described were those patients who intentionally killed themselves without MAID, but blurred boundaries existed between what participants considered a hastened death and if MAID was considered death by suicide. 
"That word is a huge barrier...it is a loaded word. But I don't think it helps us to pretend that our patients aren't actively taking their own lives...." MD3

Participants expressed feelings of conflict and powerlessness regarding the need to prevent suicide on the one hand and supporting patient autonomy on the other, along with assessing for suicide risk. This raises questions about how an experience was defined as suicide. Participants from each discipline considered 'suicide' a loaded word containing painful emotion and meaning:

"Because suicide is a loaded word in our culture. Some people... are angry for not trying harder...suicide has all this religious background...technically it is illegal, but what are you going to do, go arrest the person after they have killed themselves?" RN4 Many of the professionals interviewed have the understanding that suicide is illegal even though this is not true in the United States (35). The comments of many participants indicate that sometimes a patient's decision to intentionally end their life seemed justified.

"you have to intervene if someone is suicidal, ... I will always follow the law but ... I think people do have the right to kill themselves...so the law and social work values are not always in line with my personal values..." MSW2

The dilemma, MSW2 notes, comes from her professional obligation to report a patient at risk for suicide, which conflicts with her role to support patient autonomy. She identifies the difference between someone who would be considered suicidal due to depression or mental illness and someone who does not want to experience decline and dependence from illness.

2) MAID access and affordability

Participants from across professions recognized challenges associated with affordability and access. Most participants explained that MAID is not available to all hospice patients. Nurses, physicians and social workers spoke about disparities in healthcare, including high cost that often prohibits patients from pursuing MAID. 
“...the cost of the medication now is putting it out of reach for a lot of our patients...so this medication that was used early on was $\$ 280$ when the Death with Dignity law was passed...It is now \$3000.” MSW5

Many comments indicated that patients also experience challenges through the process of obtaining a prescription. These challenges presented a conflict to some professionals who felt they were placed in an unexpected position of responsibility and power over the decision to identify options or prevent suicide.

One way that professionals assist is by offering palliative sedation as an alternative to MAID if a patient cannot afford the medications. Hospice care supports and pays for medications used for palliative sedation for patients suffering from severe and refractory symptoms but do not pay for MAID medications:

"And one of the reasons why it has become a bigger issue is because the cost of medications for death with dignity is so high that patients ... are now asking us to do it for them..." MD3

The comment by MD3 points to requests from patients to help them die. However, professionals from all disciplines indicated that communication about desire for hastened death is common. Participants explained that most patients eventually died a natural death, but concern was expressed that some intentionally hasten death when access to MAID is limited. MSW7 described a patient who tried to pursue MAID but could not find a physician or assistance to complete the paperwork for the request:

“...she kept saying... I want to do this Death with Dignity... But she couldn't do the paperwork, couldn't go anywhere and didn't have a doctor who would do it for her as well." MSW7

Comments about limited access caused distress for this social worker and other participants 
who expressed feelings of powerlessness to support patients' wishes. A few participants explained finding a pharmacy able or willing to dispense the prescriptions makes it a challenge for anyone who wishes and is eligible to utilize MAID.

Several participants spoke of challenges they felt communicating with patients asking about MAID but did not always know if the policy of their employer forbade participation. Participants working in the organizations designated as non-profit and not religiously affiliated reported that employers had policies indicating open participation with patients who choose MAID. When two or more participants were interviewed from the same for-profit or religiously-affiliated organizations, each reported a different understanding of policy. Some said their employer influenced the degree to which they could educate patients, limiting options if patients do not have or are not given access to information about MAID.

"And we have a policy at our organization...we are not allowed to discuss these options with our patients." RN4

The comment by RN4 demonstrates potential dilemmas patients and their families may face if no healthcare professionals are present. Openly communicating about patients' choices is not always supported in the organization and may limit what is shared with other team members. Participants suggested that despite organizational policies, MAID cannot be ignored in their effort to provide care. RN7 highlights how some patients do not involve hospice professionals as they are going through the process of obtaining MAID medications, but then call for assistance after the death:

"He actually did not even let us know when he was going to do it. He just one evening said I am done, and took the medications and afterwards the wife called the hospice nurse" RN7

3) How patients have hastened their own death 
Participants shared stories about how patients intentionally hastened death or died by suicide that were not with MAID. Patients were said to have 'hastened death' by an intentional overdose of medications, either obtained illegally or saved from previous prescriptions. These hastened deaths are distinguished from suicide and suggests that the intention of the patient to die received social support or that their actions were not due to what the professional considered to be a psychopathology. For example, the death of a patient described by MSW5, like many other patients, was not identified as a suicide by the hospice team or the coroner:

“...the pharmacy didn't have his medication...and at that point in time, he was frustrated with the whole process and took all of his morphine and all of his Lorazepam that was in the home and became unconscious and died." MSW5

Voluntary stopping of eating and drinking (VSED) was discussed by most participants as another way patients sought to hasten death. This was unrelated to access to MAID in most of the interviews. Participants did not identify VSED as a form of suicide but acknowledged that hospice patients might have hastened death by days to several months using this method.

"She said I am going to stop eating and drinking on a certain date, and she said I want to know that you will be there to support me ... We didn't have permission to speak openly ... [in team meetings].” MSW7

Most of the participants from all the disciplines thought that patients who voluntarily stopped eating and drinking had control over their deaths. Staff who work with a patient sometimes feel emotionally isolated from professional scrutiny, but support the patient's decision independently from the team, as explained by MD1:

"I remember being just a little uncomfortable ... but the bottom line is as her doctor I am there to support her ... in her decisions." MD1

Most participants representing each discipline explained that they supported the patient's decision despite personal discomfort. There were several statements referring to a level of trust 
patients had with the staff member and what it takes to care for those dying. In some comments, it is suggested that VSED is slightly more acceptable to professionals and within organizations than MAID or suicide, but a cultural taboo may remain if some feel they cannot speak openly about these experiences.

Patients who killed themselves with a gun or other violent method where cause of death was obvious were identified as a suicide. Nurses, social workers and chaplains shared several traumatic accounts of arriving at a patient's home to find they had died by gunshot wound, and in some cases the patient had not yet died.

“... and we had one person who attempted to shoot themselves ... That was in hospice in a home. He missed." CH3

In most of these comments, participants said that although they did not identify the patient as a suicide risk, they regarded them as valuing independence and autonomy. Participants emphasized that despite efforts to reduce the risk of a violent death, patients still die violently by suicide from guns.

"I am not sure he trusted us in hospice ... he shot himself in the head. He left a suicide note ... there was no depression notable leading up to his suicide." MSW4

MSW4 felt that the patient had not previously demonstrated suicidal ideation or intent. However, in the following case the risk was apparent:

“... he was the one who said 'I wouldn't let my dog go through this. I feel I deserve the same.' ... shot and killed himself ... I talked to him about alternate methods. But he wasn't having it." MSW7

This statement indicates that both she and the family were aware of the patient's risk of suicide, were unable to prevent it, and were accepting of the patient's decision. But the social worker 
implied that despite her efforts to counsel the patient, there were also no structured ways to care for the patient and prevent this violent death.

\section{Discussion}

This study found hospice professionals have experiences with patients who have died by suicide and that some of these experiences are related to access to MAID. In addition, this study found that hospice professionals do not easily identify patient deaths as suicide for several reasons. Using the term 'suicide' tends to have negative connotations not only on possible emotional consequences for surviving family members but may cause increased financial burden and impact life insurance (36). Use of different language softens the reaction and experience for those who do not feel suicide or hastening of death should be prohibited in all cases.

An intentionally hastened death is described by participants as taboo when it is called suicide, making it difficult to have open discussions among team members. Participants felt that suicide assessment, prevention and treatment require a careful examination of different approaches applicable for individuals suffering with a debilitating illness. Professionals identified suicide deaths as those involving calculated plans such as patients who died by self-inflicted gunshot wounds, but even some of these deaths are considered rational or seemed justified. Patients who died by intentional overdose of medications are not consistently identified as suicide, even when family members and other healthcare workers were aware of the plan, but may nevertheless be called a hastened death. Deaths utilizing MAID are not generally considered suicide by hospice professionals but trigger reflection on what suicide means in a hospice context.

MAID has impacted professionals' experiences of suicide because there are instances when 
patients are not eligible for, cannot afford, do not have the information about or access to legal options. Palliative sedation is seen as an alternative for those who cannot afford MAID, yet this approach leaves a question whether it is considered a hastened death. If the definition of palliative sedation is "titration of opioids to symptom level at the end of life" as some participants implied, then Sheahan (37) suggests this would not be considered a hastened death if this is not the clinician's intent.

Patients are hastening their deaths in ways that are sometimes violent and can lead to uncertain consequences. Having to face the choice between MAID or dying by a violent suicide was reported by participants in this study as an inhumane solution for patients at the end of life. Participants acknowledged a profound lack of affordable care options for patients facing loss of autonomy or feelings of being a burden, important issues for those seeking MAID (38). The overall findings suggest that having safe, legal and accessible options are felt to give some patients who have knowledge and information about MAID an element of power over their lives that, paradoxically, may minimize their desire to die and encourage them to utilize palliative care (39).

\section{Strengths and limitations}

This is the first qualitative study about suicide, hastened death, and MAID that raises the unheard voices of home hospice professionals. It adds to research evidence that suggests there is a need for increased education and training regarding MAID (40-42), suicide assessment in palliative and hospice care, and open communication about issues of suicide among hospice team members $(29,43,44)$. This study supports previous research that indicates professionals do not abandon patients who seek to hasten death particularly when working in home settings $(45,46)$. This is also the first study addressing suicide in home hospice settings where MAID is allowed; previous studies have not included legal MAID $(43,47,48)$. 
Participants were aware that the researcher had worked as a hospice professional in Washington State and this may have been both a strength and a limitation. Although this study specifically explored experiences there is a benefit in balancing findings with a broader quantitative overview that may include measuring incidences of patient suicide or addressing the specific question of desire for hastened death among a wider population of hospice professionals. Further insights could be gained by speaking with professionals about how they worked to alleviate patients' suffering or to better understand the emotional impact of these experiences on team members. Future research could include a comparison of the desire for hastened death with actual deaths by suicide in palliative care settings where aid in dying is and is not lawful.

\section{Conclusion}

This study's objective was to explore hospice professionals' experiences of patients who die by suicide or intentionally hasten death with or without legal assistance in an area where MAID is legal. The reflections of professionals in this research study indicate that some patients receiving hospice services die by suicide. Hastened death is not only about MAID, but the reality of MAID offers an opportunity for dialogue about suicide and hastened death, access to care, palliative care, and safe options for the few individuals who seek to control timing of death. 


\section{Disclosures and acknowledgments}

\section{Authorship}

Concept and development: SMG, AB

Drafting: SMG, NP, AB

Review and approval of final draft: SMG, NP, AB

\section{Funding}

The authors have no financial relationship relevant to this article to disclose

\section{Disclosures and Declaration of Conflict of interest Statement}

This article originated from doctoral research completed in 2018. The authors received no funding for this study and each of the authors declare they have no conflict of interest relevant to this article.

\section{Research ethics and patient consent}

The protocol was reviewed by the Lancaster University Faculty of Health and Medicine Research Ethics Committee and approved by the Lancaster University Research Ethics Committee, FHMREC14031

\section{Acknowledgements}

The authors are grateful to Anne Grinyer, $\mathrm{PhD}$ for her contribution, advice and support during the entire period of this study. We also thank the individuals who agreed to be interviewed and the many patients they served. 
1. Emanuel EJ, Onwuteaka-Philipsen BD, Urwin JW, Cohen J. Attitudes and practices of euthanasia and physician-assisted suicide in the United States, Canada, and Europe. JAMA 2016; 316: 79-90. DOI: 10.1001/jama.2016.8499.

2. Parliament of Victoria. Voluntary Assisted Dying Act 2017

http://www.legislation.vic.gov.au/Domino/Web_Notes/LDMS/PubStatbook.nsf/f932b66241e cf1b7ca256e92000e23be/B320E209775D253CCA2581ED00114C60/\$FILE/17061aa\%20authorised.pdf $(2017,61)$.

3. California Legislative Information. SB-128, End of life Options Act, https://leginfo.legislature.ca.gov/faces/billCompareClient.xhtml?bill_id=201520160SB128 (2015).

4. Colorado Revised Statute. End of Life Options Act, http://www.sos.state.co.us/pubs/elections/Initiatives/titleBoard/filings/20152016/145Final.pdf (2016).

5. Council of the District of Columbia. Death with Dignity Act of 2016. In: Issuances OoDaA, (ed.). Washington D.C.2016.

6. Montana Supreme Court. Baxter v. State of Montana, http://cases.justia.com/montana/supreme-court/2009-12-31-DA\%20090051\%20Published\%20--\%20Opinion.pdf?ts=1396129594 (2009).

7. Oregon Legislature. The Oregon Death with Dignity Act, 127.800, https://www.oregonlegislature.gov/bills_laws/ors/ors127.html (1994).

8. Vermont General Assembly. Patient Choice At End Of Life, Vermont Statute, Title 18, Chapter $113 \mathrm{http}: / /$ legislature.vermont.gov/statutes/section/18/113/05281 (2013).

9. Washington State Department of Health. Washington State Department of Health 2015 Death with Dignity Act Report Executive Summary. In: Washington State Department of Health, (ed.). Washington2016.

10. State of New Jersey. A1504. 218th Legislature ed. 2019.

11. Death With Dignity National Center. Death with Dignity Acts, http://www.deathwithdignity.org/advocates/national (2019).

12. Al Rabadi L, LeBlanc M, Bucy T, et al. Trends in Medical Aid in Dying in Oregon and Washington. JAMA Network Open 2019; 2: e198648-e198648. DOI:

10.1001/jamanetworkopen.2019.8648.

13. Battin MP. Death with Dignity': is it suicide. OUPBlog, Oxford University Press. 2015. https://blog.oup.com/2015/11/death-with-dignity-suicide.

14. Macleod RD, Wilson DM and Malpas P. Assisted or hastened death: the healthcare practitioner's dilemma. Glob J Health Sci 2012; 4: 87-98. 11/06. DOI: 10.5539/gjhs.v4n6p87 $10.5539 /$ gjhs.v4n6p87.

14. Richards N. Old age rational suicide. Sociology Compass 2017; 11. DOI: 10.1111/soc4.12456.

16. Tierney TF. The governmentality of suicide: Peuchet, Marx, Durkheim, and Foucault. Journal of Classical Sociology 2010; 10: 357-389. DOI: 10.1177/1468795X10379677.

17. Vamos MJ. Physician-assisted suicide: saying what we mean and meaning what we say. Aust N Z J Psychiatry 2012; 46: 84-86. DOI: 10.1177/0004867411432069.

18. Werth JL and Holdwick DJ. A primer on rational suicide and other forms of hastened death. Counseling Psychologist 2000; 28: 511-539. DOI: 10.1177/0011000000284003.

19. Gerson SM, Bingley A, Preston N, Grinyer A. When is hastened death considered suicide? A systematically conducted literature review about palliative care professionals' experiences where assisted dying is legal. BMC palliative care. 2019 Dec;18(1):1-3. DOI: $\underline{10.1186 / \mathrm{s} 12904-019-0451-4 .}$. 
20. Bostwick JM and Cohen LM. Differentiating suicide from life-ending acts and endof-life decisions: A model based on chronic kidney disease and dialysis. Psychosomatics 2009; 50: 1-7. DOI: 10.1176/appi.psy.50.1.1.

21. Back AL, Starks H, Hsu C, et al. Clinician-patient interactions about requests for physician-assisted suicide: a patient and family view. Arch Intern Med 2002; 162: 12571265. DOI: 10.1001/archinte.162.11.1257.

22. Cohen JS, Fihn SD, Boyko EJ, Jonsen AR, Wood RW. Attitudes toward assisted suicide and euthanasia among physicians in Washington State. N Engl J Med 1994; 331: 8994. 07/14. DOI: 10.1056/nejm199407143310206.

23. Emanuel EJ, Daniels ER, Fairclough DL, Clarridge BR. The practice of euthanasia and physician-assisted suicide in the United States: adherence to proposed safeguards and effects on physicians. JAMA 1998; 280: 507-513. 08/26. DOI: 10.1001/jama.280.6.507. 24. Galushko M, Frerich G, Perrar KM, et al. Desire for hastened death: how do professionals in specialized palliative care react? Psycho-Oncology 2016; 25: 536-543. DOI: 10.1002/pon.3959.

25. Magnusson RS. Angels of death: exploring the euthanasia underground. Yale University Press, 2002.

26. Starks H, Pearlman RA, Hsu C, et al. Why now? Timing and circumstances of hastened deaths. J Pain Symptom Manage 2005; 30: 215-226. DOI:

10.1016/j.jpainsymman.2005.03.012.

27. Stevens CA and Hassan R. Management of death, dying and euthanasia: attitudes and practices of medical practitioners in South Australia. J Med Ethics 1994; 20: 41-46. 03/01. DOI: $10.1136 / \mathrm{jme}$.20.1.41.

28. Tamayo-Velázquez M-I, Simón-Lorda P and Cruz-Piqueras M. Euthanasia and physician-assisted suicide: Knowledge, attitudes and experiences of nurses in Andalusia (Spain). Nursing Ethics 2012; 19: 677-691. DOI: 10.1177/0969733011436203.

29. Warren SC and Zinn CL. Review of completed suicides in a community hospice. Journal of palliative medicine 2010; 13: 937-938. DOI: 10.1089/jpm.2010.0107.

30. Oliver DP, Wittenberg-Lyles E, Washington K, et al. Hospice caregivers' experiences with pain management:"I'm not a doctor, and I don't know if I helped her go faster or slower”. J Pain Symptom Manage 2013; 46: 846-858. DOI:

10.1016/j.jpainsymman.2013.02.011.

31. Ong J, Brennsteiner A, Chow E, Hebert RS. Correlates of family satisfaction with hospice care: general inpatient hospice care versus routine home hospice care. Journal of palliative medicine 2016; 19: 97-100. DOI: 10.1089/jpm.2015.0055.

32. Campbell CS and Black MA. Dignity, death, and dilemmas: a study of Washington hospices and physician-assisted death. J Pain Symptom Manage 2014; 47: 137-153. DOI: 10.1016/j.jpainsymman.2013.02.024.

33. Braun V and Clarke V. What can "thematic analysis"'offer health and wellbeing researchers? Int J Qual Stud Health Well-being 2014; 9. DOI: 10.3402/qhw.v9.26152.

34. Braun V, Clarke V. Successful Qualitative Research: A practical guide for beginners. London: Sage. 2013.

35. Mishara BL and Weisstub DN. The legal status of suicide: A global review. Int J Law Psychiatry 2016; 44: 54-74. DOI: 10.1016/j.ijlp.2015.08.032.

36. Young IT, Iglewicz A, Glorioso D, et al. Suicide bereavement and complicated grief. Dialogues Clin Neurosci 2012; 14: 177-186.

37. Sheahan L. Exploring the interface between 'physician-assisted death'and palliative care: cross-sectional data from Australasian palliative care specialists. Internal medicine journal 2016; 46: 443-451. DOI: doi.org/10.1111/imj.13009.hsa 
38. Lee BC and New York Acad S. Oregon's experience with aid in dying: findings from the death with dignity laboratory. Annals of the New York Academy of Sciences 2014; 1330(1) 94-100. DOI: 10.1111/nyas.12486.

39. Wang S-Y, Aldridge MD, Gross CP, et al. Geographic variation of hospice use patterns at the end of life. Journal of palliative medicine 2015; 18: 771-780.

DOI: $10.1089 / \mathrm{jpm} .2014 .0425$.

40. Gamondi C, Borasio GD, Oliver P, Preston N, Payne S. Responses to assisted suicide requests: an interview study with Swiss palliative care physicians. BMJ Supportive \& Palliative Care 2017: bmjspcare-2016-001291. DOI: 10.1136/bmjspcare-2016-001291. 41. Otte IC, Jung C, Elger B, Bally K. "We need to talk!” Barriers to GPs' communication about the option of physician-assisted suicide and their ethical implications: results from a qualitative study. Medicine, Health Care and Philosophy 2016: 1-8. DOI: 10.1007/s11019-016-9744-z.

42. Pasman HR, Willems DL and Onwuteaka-Philipsen BD. What happens after a request for euthanasia is refused? Qualitative interviews with patients, relatives and physicians. Patient education and counseling 2013; 92: 313-318. 2013/07/09. DOI:

10.1016/j.pec.2013.06.007.

43. Fairman N, Montross Thomas LP, Whitmore S, Meier E, Irwin S. What did I miss? A qualitative assessment of the impact of patient suicide on hospice clinical staff. Journal of palliative medicine 2014; 17: 832-836. DOI: 10.1089/jpm.2013.0391.

44. Grzybowska P and Finlay I. The incidence of suicide in palliative care patients. Palliat Med 1997; 11: 313-316. DOI: 10.1177/026921639701100408.

45. Giordano J. Hospice, Palliative Care, and Pain Medicine: Meeting the Obligations of Non-Abandonment and Preserving the Personal Dignity of Terminally Ill Patients. Del Med J 2006; 78: 419-422.

46. Periyakoil VS. First, do not abandon. Journal of palliative medicine. 2007 Aug 1;10(4):865-6. DOI: 10.1089/jpm.2007.9932.

47. Arnold EM. Factors that influence consideration of hastening death among people with life-threatening illnesses. Health Soc Work 2004; 29: 17-26. DOI: 10.1093/hsw/29.1.17. 48. Washington KT, Albright DL, Oliver DP, et al. Hospice and palliative social workers' experiences with clients at risk of suicide. Palliative and Supportive Care 2016: 1-8. DOI: 10.1017/S1478951516000171. 